\title{
A Robust Controller for the Manipulation of Micro- Scale Objects
}

\author{
S. Jagannathan and Qinmin Yang
}

\begin{abstract}
A suite of novel robust controllers is presented for the manipulation and handling of micro-scale objects in a micro-electromechanical system (MEMS) where adhesive, surface tension, friction and van der Waals forces are dominant. Moreover, these forces are typically unknown. The robust controller overcomes the unknown system dynamics and ensures the performance in the presence of actuator constraints by assuming that the upper bounds on these forces are known. On the other hand, for the robust adaptive controller, the unknown forces are estimated online. Using the Lyapunov approach, the uniformly ultimate boundedness (UUB) of the closed-loop manipulation error is shown for pick and place tasks. Simulation results are presented to substantiate the theoretical conclusions.
\end{abstract}

\section{INTRODUCTION}

$\mathrm{M}$ ICRO-electromechanical system (MEMS) is a relatively new technology involving miniaturization of systems and components to create complex machines that are of micron size in nature. These are used in a variety of applications involving sensing, actuation and communication. The MEMS has revolutionized a major part of the sensor and actuator industry. Modeling of such micro-scale devices for actuation is a whole lot different from that in macro scale system. At micro scale, surface forces are predominant while volumnic forces are negligible [1]. The dominant forces acting on a MEMS system are adhesive, electrostatic and van der Waals forces, while the forces due to gravity are negligible. Uncertainties, for instance fabrication imperfections and complex system nonlinearities, make the actuation and manipulation of such devices difficult.

Designing controllers for the manipulation and handling of micro-scale objects poses a much greater challenge in terms of accommodating the nonlinearities in the system. Hence, these forces have to be modeled in order to design a controller for the micro system. To confront some of the issues of nonlinearities and uncertainties in such MEMS systems, a robust controller is designed. The robust controller requires the upper bound on the uncertainties and nonlinearities. Actuator constraints have been incorporated into this controller. By contrast, in the case of robust

The research is supported in part by NSF grant ECS \# 0296191 and Intelligent Systems Center.

S. Jagannathan and Qingming Yang are with the Department of Electrical and Computer Engineering, University of Missouri-Rolla, MO 65409 , USA. adaptive neural network (NN) controller, reinforcement learning (RLNN) feature [11] is utilized to approximate the uncertainties online. The RLNN-based controller has two NNs: an action NN for compensating the uncertain nonlinear system dynamics, and a critic NN for tuning the action NN weights. A novel utility function, which is viewed as the system performance index over time, was defined as the critic NN input. The critic signal provides an additional corrective action based on current and past system performance. This information along with the filtered tracking error is used to tune the action NN.

\section{MODELING}

Manipulation and handling of micro-scale objects are required for the assembly and maintenance of micro machines and their parts. In this study, we consider the manipulation of micro-sized spheres 50 $\mu \mathrm{m}$ in diameter. When manipulating objects in the micro domain, the microphysics of the problem must be taken into account [2], [3]. Modeling is necessary for picking up micro-spheres laying on a planar substrate. The micro-sphere is to be picked up and it needs to be placed at another location. The probe, which is treated as an end-effector and manipulator, is lowered to make contact with the micro-sphere. Once contact has been established, the probe is to be retracted and the micro-object has to be picked up due to the adhesive forces [4].

The process of placing the micro-object after it has been picked- up is also an intricate process. For the purpose of designing a controller for the object-handling task, we shall restrict ourselves with the intricacies of the physics of the picking up process as shown in Fig. 1. Hence, the adhesion forces are dominant in the system. Adhesive forces considered to play an important role in the manipulation process are:

- Van der Waals forces,

- Electrostatic forces (or coulomb) and

- Surface tension (or capillary) forces.

Materials and object geometries greatly decide the magnitude of van der Waals forces. For ideal geometries the van der Waals forces are given by

$$
F_{b p}^{V d W}=\frac{A_{b p}^{w} R_{b}}{6 D_{b p}^{2}}, F_{b s}^{V d W}=\frac{A_{b s}^{w} R_{b}}{6 D_{b s}^{2}}, \text { and } F_{b b}^{V d W}=\frac{A_{b b}^{w} R_{b}}{6 D_{b b}^{2}},
$$

respectively for ball-probe, ball-substrate and for ball-ball interaction. $R_{b}$ is the sphere radius, $A_{i j}^{w}$ is the Hamaker 
constant of " $\mathrm{i}$-water-j" interface, and $D_{i j}$ is the separation distances. Van der Waals forces are greatly influenced by the surface roughness [2]. It has been shown that increasing the surface roughness decreases the van der Waals forces [4]. Taking the surface roughness into consideration as shown in Fig. 2, the van der Waals force is given by [6]

$$
F_{v d w b}=\left(\frac{z}{z+b / 2}\right)^{2} F_{v d w},
$$

where $z$ is the distance, $b$ is the height of the surface irregularities, and $F_{v d w}$ is the van der Waals forces between the plane plate and the plane sphere.

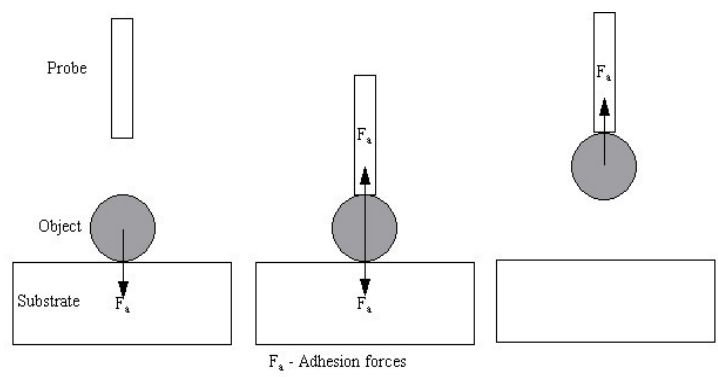

Fig. 1. Object handling task.

The capillary or surface tension forces are expressed as

$$
F_{b p}^{c a p}=\frac{4 \pi \gamma R_{b} d}{d+D_{b p}}, F_{b s}^{c a p}=\frac{4 \pi \gamma R_{b} d}{d+D_{b s}} \text { and }{ }_{F_{b b}}^{c a p}=\frac{4 \pi \gamma R_{b} d}{d+D_{b b}},
$$

respectively for the ball-probe, ball-substrate and the ballball interactions. The variables $\gamma$ and $d$ denote the surface tension and the height of immersion respectively.

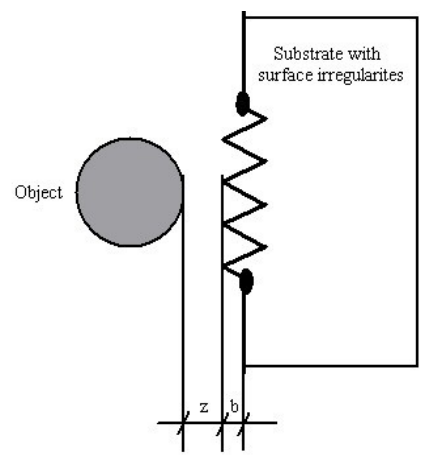

Fig. 2. Rough plate and plane sphere.

The electrostatic force between an uncharged metal wall and a charged sphere is given by

$$
F_{e}=\varepsilon_{0} \pi d^{2}\left(\frac{3 \varepsilon_{1}}{\varepsilon_{1}+2}\right)^{2} E^{2},
$$

where $\varepsilon_{0}$ and $\varepsilon_{1}$ are the dielectric constants of free-space and the material, respectively. The parameter, $d$, is the reduced diameter obtained as $d=\frac{d_{1} d_{2}}{d_{1}+d_{2}}$, where, $d_{1}$ and $d_{2}$ are the diameters of the two spheres under consideration. The parameter, $E$, is the voltage between the probe and the substrate. It has also been shown that the electrostatic forces can be minimized by applying an external voltage.

\section{DYNAMIC MODEL}

A dynamic model of the micro-scale object handling system is formulated considering all the forces mentioned above [4], [7]. The objects considered in this work include micro-spheres of diameter 50 to $200 \mu \mathrm{m}$ (radius $R_{b}$ varies from $25 \mu \mathrm{m}$ to $100 \mu \mathrm{m}$ ).

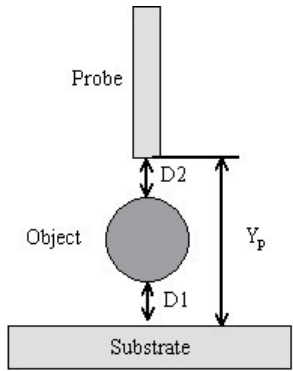

a)

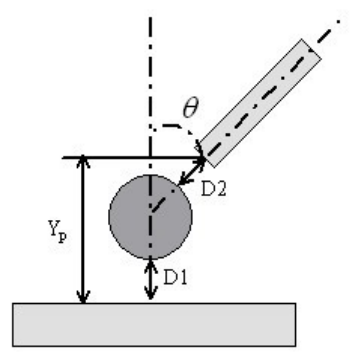

Fig. 3. Intersurfacic distances notation a) capture at straight and $b$ ) capture at an inclination.

The dynamic model for the object handling task is [4] $m_{p} \ddot{Y}_{p}=F_{e x t} \cos \left(\frac{\pi}{2}-\theta\right)-F_{b p}^{V d W} \cos \theta-F_{b p}^{c a p} \cos \theta-F_{b p}^{e l e c} \cos \theta-m_{p} g$,

$m_{b} \ddot{D}_{1}=\left(F_{b p}^{V d W}+F_{b p}^{c a p}+F_{b p}^{\text {elec }}\right) \cos \theta-F_{b s}^{V d W}-F_{b s}^{c a p}-F_{b s}^{\text {elec }}-m_{b} g$,

$$
Y_{p}=D_{1}+R_{b}+\left(R_{b}+D_{2}\right) \cos \theta,
$$

where $\ddot{Y}_{p}$ is the instantaneous acceleration of the probe, $F_{\text {ext }}$ is the external force applied to the probe, $\theta$ is the angle of inclination of the probe with the vertical axis, $F_{i j}^{V d W}$ is the van der Waals forces, $F_{i j}^{c a p}$ is the capillary forces and $F_{i j}^{e l e c}$ is the electrostatic forces for the ballprobe (bp) and the ball-substrate (bs) interfaces presented in (1) through (8) respectively. There are two constraints for this model

- a condition imposed by the substrate reaction (when ball contacts the substrate at $\left.D_{1}=D_{0}=0.4 \mathrm{~nm}\right)$ :

$$
D_{1}=0.4 n m \Rightarrow \ddot{D}_{1} \geq 0 .
$$

- a detachment constraint expressed by:

$$
F_{\text {ext }}>2 R_{b} \pi W_{\text {ball-water-substrate }} \text {, }
$$

where $W_{\text {ball-water-substrate }}$ is the surface work of adhesion. 
The manipulation time has to be small and applied force has to be appropriate to prevent ball substrate deformation. The dynamic model for the manipulation and handling of micro-scale objects are quite nonlinear and unknown. For instance, the surface tension, Hamaker constant, electric charge density, diameter of the object, height of immersion and so on is typically unknown. Under these circumstances, one has to apply advanced control schemes in order to manipulate such micro-scale objects. The control scheme must guarantee object manipulation in the event of such unknown uncertainties.

\section{Controller Design}

The suite of controller designs proposed in this paper is based on the filtered tracking error formulation. The error system formulation along with the robust controller and robust adaptive neural network controller has been discussed in detail. For the purpose of controller design, $\theta$ is considered as constant.

\section{A. Filtered Tracking Error Dynamics Formulation}

The manipulation and handling of the sphere is done when $D_{1}$ increases and $D_{2}=D_{0}$ (atomic contact distance). On the other hand, for placing the objects on a substrate at a different location, $D_{1}$ must be equal to the atomic contact distance whereas $D_{2}$ must be increasing. Depending upon whether picking up the object or placement of the object, the control objective is suitably chosen as mentioned above. However, the controller development is the same whether the object is picked or placed. Therefore, here we present the controller development using the object pick up. Differentiating (8), to get

$$
\dot{Y}_{p}=\dot{D}_{1}+\dot{D}_{2} \cos \theta
$$

and

$$
\ddot{Y}_{p}=\ddot{D}_{1}+\ddot{D}_{2} \cos \theta
$$

or

$$
\ddot{D}_{2}=\frac{1}{\cos \theta}\left(\ddot{Y}_{p}-\ddot{D}_{1}\right),
$$

Let the error $e$ between the desired and the target position be defined as

$$
e=D_{2}-D_{0}
$$

Thus, when the error goes to zero, then $D_{2}=D_{0}$ and the probe picks up the micro-sphere. Differentiating (13) to get:

$$
\dot{e}=\dot{D}_{2}
$$

and

$$
\ddot{e}=\ddot{D}_{2}=\frac{1}{\cos \theta}\left(\ddot{Y}_{p}-\ddot{D}_{1}\right),
$$

Let $r$ be the filtered tracking error which is defined as,

$$
r=\dot{e}+\Lambda e \text {, }
$$

where $\Lambda \in R$ is a positive design parameter.

Differentiating (16) to get

$$
\dot{r}=\ddot{e}+\Lambda \dot{e},
$$

Substituting for $\ddot{e}$ and $\dot{e}$ from (15) and (14) results in

$$
\dot{r}=\frac{1}{\cos \theta}\left(\ddot{Y}_{p}-\ddot{D}_{1}\right)+\Lambda \dot{D}_{2}=\left(F\left(Y_{p}\right)-F\left(D_{1}\right)\right)+\Lambda \dot{D}_{2}+v
$$

where

$$
F\left(Y_{p}\right)=\frac{1}{m_{p}}\left(-F_{b p}^{V d W}-F_{b p}^{c a p}-F_{b p}^{\text {elec }}-\frac{1}{\cos \theta} m_{p} g\right),
$$

and

$$
F\left(D_{1}\right)=\frac{1}{m_{b}}\left(F_{b p}^{V d W}+F_{b p}^{c a p}+F_{b p}^{e l e c}\right)-\left(F_{b s}^{V d W}+F_{b s}^{c a p}+F_{b s}^{e l e c}+m_{b} g\right) \frac{1}{m_{b} \cos \theta},
$$

and $v$ is the control input given by

$$
v=\frac{1}{m_{p} \cos \theta} F_{e x t} \cos (\pi / 2-\theta)=\frac{\tan \theta}{m_{p}} F_{e x t},
$$

or

$$
\dot{r}=F(X)+\Lambda \dot{D}_{2}+v,
$$

where $_{F(X)=F\left(Y_{p}\right)-F\left(D_{1}\right)}$ is an unknown nonlinear function.

\section{B. Robust Controller Design}

A robust saturation controller can be selected as

$$
v=-\hat{F}(X)-\Lambda \dot{D}_{2}-k_{v} r-v_{1},
$$

where $k_{v} \in R$ is the feedback gain and the auxiliary feedback signal is chosen later with $\hat{F}(X)$ is an estimate for $\mathrm{F}(\mathrm{X})$ that is not changed online.

Assumption 1: Let $F_{M}(X)$ is a known scalar function that bounds the uncertainties $\tilde{F}(X)=F(X)-\hat{F}(X)$ so that

$$
\|\tilde{F}(X)\| \leq F_{M}(X) \cdot
$$

The intent is that $F_{M}(X)$ is a simplified function that can be computed using the bounding properties of the forces that act upon the microsphere. Observing the microforces, it can be seen that the forces are upper bounded.

Applying (24) in (22) to get

$$
\dot{r}=-k_{v} r+(F(X)-\hat{F}(X))+v_{1},
$$

or

$$
\dot{r}=-k_{v} r+\tilde{F}(X)+v_{1}
$$

When $\hat{F}(X)$ is an accurate estimate of $F(X)$ and in the presence of no auxiliary signal, then $\tilde{F}(X) \rightarrow 0$ and (28) becomes

$$
\dot{r}=-k_{v} r
$$

If $k_{v}$ is properly selected as a positive constant, then from (29) and (16) one can see that $e \rightarrow 0$ with $t \rightarrow \infty$. Thus, $D_{2}=D_{0}$ and the sphere is said to be manipulated (pick-up task). 
In order to incorporate the magnitude constraints with the controller, now select $\Delta u=v-\tau$ or $v=\tau+\Delta u$ where $v$ is the control input and $\tau$ is the applied force, which is selected to be equal to (24). Applying the magnitude constraints on the control input, we have $v \quad=\tau \quad$ for $|\tau(t)| \leq v_{\max }$ $=v_{\max } \operatorname{sgn}(v(t)) \quad$ for $|\tau(t)|>v_{\text {max }}$

Equation (22) now results in $\dot{r}=-k_{v} r+\tilde{F}(X)+v_{1}+\Delta u$ where $\Delta u$ is defined as a disturbance. In order to combat disturbance, define $\dot{e}_{\Delta}$ as

$$
\dot{e}_{\Delta}=-k_{v} e_{\Delta}+\Delta u \text {. }
$$

Now define the error as

$$
e_{u}=r-e_{\Delta} .
$$

Differentiating (31) and substituting (30) in (31) to get

$$
\dot{e}_{u}(t)=-k_{v} e_{u}(t)+\tilde{F}(X)+v_{1},
$$

Select the auxiliary input as [7]

$$
v_{1}=\left\{\begin{array}{l}
-e_{u} \frac{F_{M}(X)}{\left|e_{u}\right|},\left|e_{\mathrm{u}}\right| \geq \beta, \\
-e_{u} \frac{F_{M}(X)}{\beta},\left|e_{\mathrm{u}}\right|<\beta
\end{array}\right.
$$

when $|\tau(t)| \leq v_{\text {max }}$ and otherwise, set the auxiliary input, $v_{1}$, to be zero. In computing the robust control term $v_{1}, \beta$ is a small design parameter.

Theorem 1: Consider the system given in (5), (6) and (7), and take the Assumption 1. Then using the robust controller (29), the error, $\left|e_{u}\right|,|r|$ and $|e|$ is eventually bounded to the neighbourhood of $\beta$.

Proof: We will take the case when $|\tau(t)| \leq v_{\max }$. Select the Lyapunov function candidate

$$
L=\frac{1}{2} \mathrm{e}_{\mathrm{u}}^{2} \text {. }
$$

Differentiate the above equation and substituting error dynamics (32) to get

$$
\begin{aligned}
\dot{L} & =-k_{v} e_{u}^{2}+e_{u} \tilde{F}(X)+e_{u} v_{1} . \\
& \leq-k_{v} e_{u}^{2}+\left|e_{u}\right| F_{M}(X)+e_{u} v_{1} .
\end{aligned}
$$

There are now two cases to consider- $\left|e_{u}\right| \geq \beta$ and $\left|e_{u}\right|<\beta$.

Case 1. $\left|e_{u}\right| \geq \beta$. In this case, according to the definition of the robust control term (33), one has

$$
\begin{aligned}
\dot{\mathrm{L}} & \leq-k_{v} e_{\mathrm{u}}{ }^{2}+\left|e_{u}\right| F_{M}(X)-e_{u}{ }^{2} F_{M}(X) /\left|e_{u}\right| \\
& \leq-k_{v} e_{\mathrm{u}}{ }^{2} .
\end{aligned}
$$

Therefore $\dot{L}$ is negative in terms of $\left|e_{u}\right|$. Hence $\mathrm{L}$ is decreasing in this region and $\left|e_{u}\right|$ decreases towards $\beta$.
Case 2. $\left|e_{u}\right|<\beta$. In this case, according to the definition of the robust control term (33), one has

$$
\begin{aligned}
\dot{\mathrm{L}} & \leq-k_{v} e_{\mathrm{u}}^{2}+\left|e_{u}\right| F_{M}(X)-e_{u}^{2} F_{M}(X) / \beta . \\
& \leq-k_{v} e_{u}^{2}+\left|e_{u}\right| F_{M}(X)\left(1-\left|e_{u}\right| / \beta\right) .
\end{aligned}
$$

The last term is generally positive in this region, so nothing can be said about whether $\mathrm{L}$ is increasing or decreasing. In general L may be increasing in this region so that $\left|e_{u}\right|$ increases towards $\beta$. Given the boundedness of $\left|e_{u}\right|$ and using (31), one can conclude $|r|$ is bounded. Using (16), $|e|$ is bounded.

Similarly the proof can be shown when $|\tau(t)|>v_{\max }$.

\section{Adaptive Neural Network Controller Design}

In the above section, a robust controller with magnitude constraints on the input is presented wherein the unknown dynamics of the manipulation system is overcome by a bounded known function. In this subsection, an adaptive neural network (NN) [11] is utilized where the unknown manipulation dynamics are approximated online.

An action $\mathrm{NN}$ is employed to approximate this unknown system dynamics. According to [12], a single layer NN can be used to approximate any nonlinear continuous function over the compact set when the input layer weights are selected at random and held constant whereas the output layer weights are only tuned provided sufficiently large number of nodes in the hidden-layer is chosen. Therefore, a single layer $\mathrm{NN}$ is employed here whose output is defined as $\hat{w}_{1}^{T} \varphi\left(v_{1}^{T} X\right)$, where $\hat{w}_{1} \in R^{n_{1}}$ and $v_{1} \in R^{4 \times n_{1}}$ are the output and input layer weights, $n_{1}$ is the number of the hidden layer nodes, $\varphi(\cdot)$ is the activation function vector, and $X=\left[Y_{p}, D_{2}, \dot{Y}_{p}, \dot{D}_{2}\right]^{T} \in R^{4}$ is the input to the neural network. For simplicity, the action $\mathrm{NN}$ output is given as

$$
\hat{F}(X)=\hat{w}_{1}^{T} \varphi(X) .
$$

Thus, a control input can be selected as

$$
v=-\hat{F}(X)-\Lambda \dot{D}_{2}-k_{v} r
$$

where $k_{v} \in R$ is the feedback gain selected to be positive constant. Applying (39) in (22) to get

$$
\dot{r}=-k_{v} r+(F(X)-\hat{F}(X)),
$$

or

$$
\dot{r}=-k_{v} r+\widetilde{F}(X),
$$

where $\tilde{F}(X)=F(X)-\hat{F}(X)$ is the function approximation error. When the neural network is properly trained and $\hat{F}(X)$ is an accurate estimate of $F(X)$, then $\tilde{F}(X) \rightarrow 0$ and (41) becomes 


$$
\dot{r}=-k_{v} r .
$$

If $k_{v}$ is properly selected as a positive constant, then from (37) and (16) one can see that $e \rightarrow 0$ with $t \rightarrow \infty$. Thus, $D_{2}=D_{0}$ and the sphere is said to be manipulated (pick-up task). Similarly, one can show the case of placement task.

The unknown function $F(X)$ can be approximated by the action $\mathrm{NN}$ as

$$
F(X)=w_{1}^{T} \varphi\left(v_{1}^{T} X\right)+\varepsilon(X)=w_{1}^{T} \varphi(X)+\varepsilon(X),
$$

where $w_{1} \in R^{n_{1}}$ is the target output layer weight, and $\varepsilon(X)$ is the NN approximation error. Define the weight estimation error $\widetilde{w}_{1} \in R^{n_{1}}$ by

$$
\widetilde{w}_{1}=w_{1}-\hat{w}_{1},
$$

Thus (40) becomes

$$
\dot{r}=-k_{v} r+\widetilde{w}_{1}^{T} \varphi(X)+\varepsilon(X) .
$$

The input to the critic NN is chosen as [11]

$$
z(t)=\int_{0}^{t} r^{2}(\tau) d \tau .
$$

A choice of the critic $\mathrm{NN}$ signal is given by

$$
R(t)=\hat{w}_{2}^{T} \sigma\left(v_{2}^{T} z(t)\right)=\hat{w}_{2}^{T} \sigma(z(t)),
$$

where $\hat{w}_{2} \in R^{n_{2}}$ and $v_{2} \in R^{n_{2}}$ are the output and input layer weights, $n_{2}$ is the number of the hidden layer nodes, $\sigma(\cdot)$ is the hidden layer activation function vector, and $z(t) \in R$ is the input to the neural network. The critic NN input defines the long term system performance over time. The critic signal, $R(t)$, provides an additional corrective action based on current and past performance. This information along with filtered tracking error is used to tune the action NN. The critic signal can also be viewed as a look-ahead factor, which is determined based on past performance. The proposed reinforcement learning-based $\mathrm{NN}$ controller structure is depicted in Fig. 4. The next step is to determine the weight updates so that the performance of the closed-loop tracking error dynamics is guaranteed. Assumption 2: The desired trajectory $\mathrm{D}_{0}$ is bounded so that $\left|D_{0}\right|<D_{B}$ with $D_{B}$ a known scalar bound. In fact, $\mathrm{D}_{0}$ becomes the inter-atomic distance.

Assumption 3: The NN approximation error $\varepsilon(X)$ is bounded above by $|\varepsilon(X)|<\varepsilon_{N}$ over the compact set.

Assumption 4: Both the ideal weights and the activation functions for all NNs are bounded by known positive values so that

$$
\begin{gathered}
\left\|w_{1}\right\| \leq w_{1 \max },\left\|w_{2}\right\| \leq w_{2 \max }, \\
\|\sigma(\cdot)\| \leq \sigma_{\max },\|\varphi(\cdot)\| \leq \varphi_{\max } .
\end{gathered}
$$

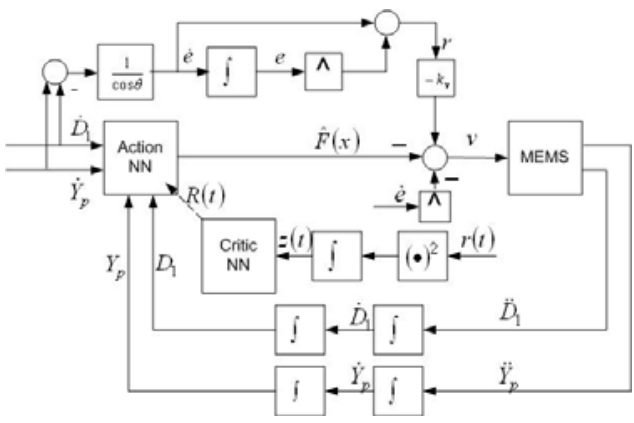

Fig. 4. Structure of the NN controller.

Theorem 2: Consider the system given in (5), (6) and (7), and take the Assumptions 2 through 4. Let the action NN weights tuning be given by

$$
\dot{\hat{w}}_{1}=\varphi(X)\left(r-\hat{w}_{1}^{T} \varphi(X)+k_{1} R(t)\right),
$$

where $k_{1}$ is a design parameter and $R(t)$ is the critic signal, which is given by the critic NN in (32). The critic NN weights be tuned by

$$
\dot{\hat{w}}_{2}=-\sigma(X)(r+R(t)) \text {, }
$$

with the control signal selected by (24). Then the filtered tracking error $r$ and the NN weights estimates, $\hat{w}_{1}$ and $\hat{w}_{2}$, are $U U B$ provided:

$$
\begin{aligned}
& \text { (1) } k_{v}>\frac{1}{2}, \\
& \text { (2) } 1>k_{1}>0 .
\end{aligned}
$$

Proof: See [11].

\section{Simulation}

It is assumed that the object is in contact with the substrate before it is picked up by the probe. The controller provides the force to cause the actual capture and to retain the micro-sphere at the tip of the probe. Once the capture occurs, and the external force to be applied through the probe is determined and maintained to keep the microsphere captured. A PD controller is designed based on the filtered tracking error.

Fig. 5 shows the distances for a proportional controller $\left(k_{v}=5, \Lambda=10^{-3}\right)$. Fig. 6 shows the control input, which appears to be highly oscillatory. Moreover, it takes longer to capture the micro-sphere. Fig. 7 shows the distances and Fig. 8 shows the control input resulting from using a reinforcement learning-based controller ( $k_{v}=5, \Lambda=10^{-3}, k_{1}=0.8$ ). It can be seen that capture occurs around $10^{-3} \mathrm{~s}$. 


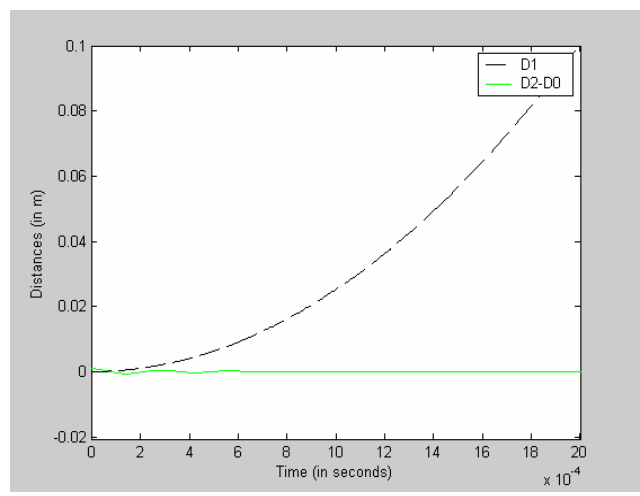

Fig. 5. Displacement using a conventional controller.

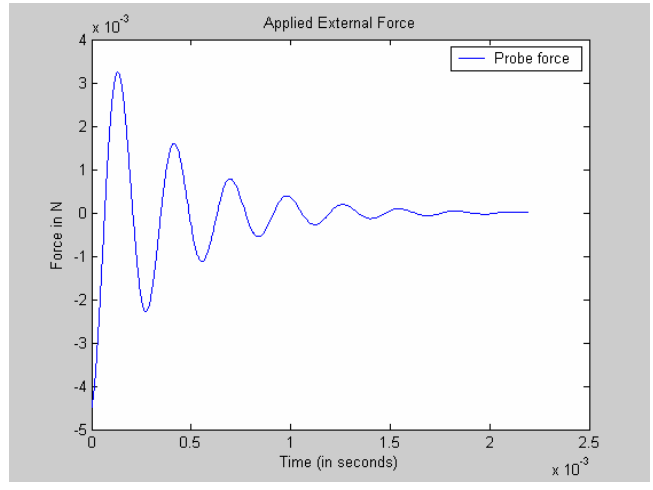

Fig. 6. Applied external force.

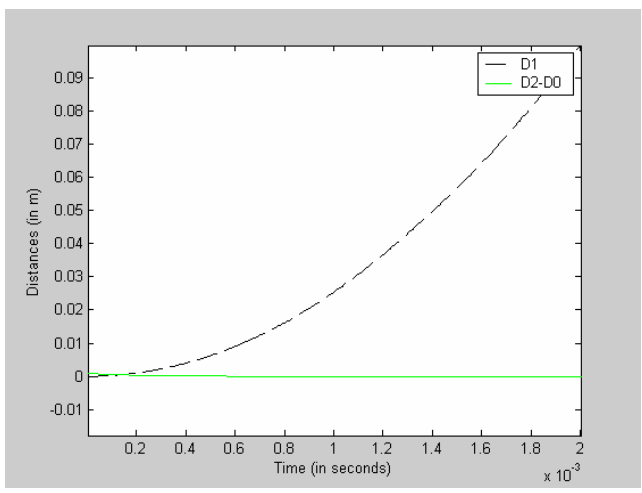

Fig. 7. Displacement with the NN controller.

\section{CONCLUSIONS}

In this paper, a suite of robust manipulation controllers was presented. Closed-loop stability in terms of manipulation of microscale objects is demonstrated using a robust controller by assuming that the upper bound on the unknown dynamics of the manipulation system is known. Then, a reinforcement learning-based adaptive $\mathrm{NN}$ controller was presented for the task of picking up a microsphere from a substrate. The controllers have been proved to have guaranteed stability and the task of manipulation was possible even when the nonlinearities and uncertainties are not modeled for. Simulation results indicate that the NN controller outperforms a conventional PD controller in terms of the response time and applied force during the object manipulation.

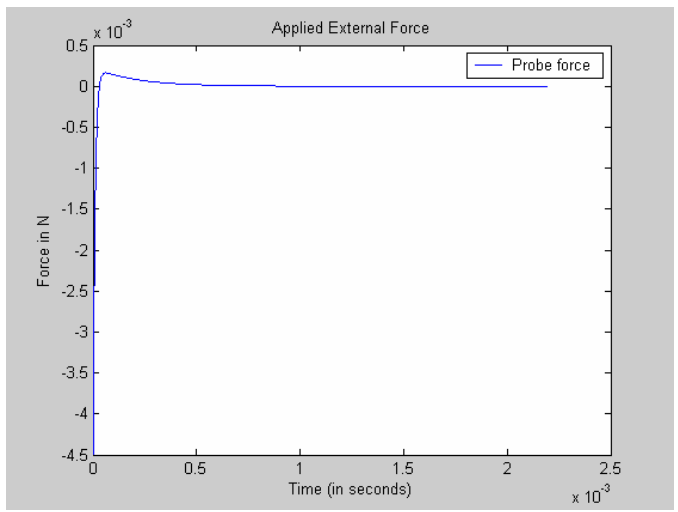

Fig. 8. Applied force using the NN controller.

\section{REFERENCES}

[1] J. T. Feddema, P. Xavier, and R. Brown "Micro-assembly planning with van der Waals force," in IEEE International Symposium on Assembly and Task Planning, pp. 32 - 38, 1999.

[2] F. Arai, D. Andou, and T. Fukuda, "Adhesion forces reduction for micro manipulation based on micro physics", Micro Electro Mechanical Systems, MEMS '96, pp.354 -359, 1996.

[3] M. Sitti, "Survey of nanomanipulation systems," in IEEE Proceedings of the Nanotechnology, pp. 75 - 80, 2001.

[4] Y. Rollot, S. Regnier, S. Haliyo, L. Buchaillot, J. C. Guinot and P. Bidaud, "Experiments on micromanipulation using adhesion forces in unconstrained environment," in Proceedings of the 2000 IEEE/RS International Conference on Intelligent Robots and Systems, vol. 1, pp. $653-658,2000$.

[5] F. Arai, D. Andou, Y. Nonoda, T. Fukuda, H. Iwata and K. Itoigawa, "Integrated micro-end effector for micromanipulation," IEEE/ASME Transactions on Mechatronics, vol. 3, pp. 17 - 23, 1998.

[6] S. Saito, H. Miyazaki, and T. Sato, "Pick and place operation of a micro-object with high reliability and precision based on microphysics under SEM," IEEE International Conference on Robotics and Automation, vol. 4, pp. 2736 - 2743, 1999.

[7] F. L. Lewis, S. Jagannathan and A. Yesilderik, Neural Network Control of Robot Manipulators and Nonlinear Systems, Taylor and Francis, 1999.

[8] P. J. Werbos, "Neurocontrol and supervised learning: An overview and evaluation", Handbook of Intelligent Control, edited by David A. White and Donald A. Sofge, Van Nostrand Reinhold, 1992, pp. 65-90.

[9] A. G. Barto, "Reinforcement learning and adaptive critic methods", Handbook of Intelligent Control, edited by David A. White and Donald A. Sofge, Van Nostrand Reinhold, pp. 469-492, 1992.

[10] B. Igelnik and Y. H. Pao, "Stochastic choice of basis functions in adaptive function approximation and the functional-link net," IEEE Trans. Neural Networks, vol. 6, 1995.

[11] V. Janardhan, P. He and S. Jagannathan, "Neural network controller for the manipulation of microscale objects", Proc. of the IEEE Symposium on Intelligent Control, 2004. 